unusual case of orbital trauma with a large foreign body in the maxilla. Br J Plast Surg 1999;52:507-8.

3. Neff LL, Liess BD, Chang CW. Transoral endoscopic removal of a bullet from the infratemporal fossa. Otolaryngol Head Neck Surg 2008; 138:113-4.

4. Grobbelaar A, Knottenbelt JD. Retained knife blades in stab wounds of the face: is simple withdrawal safe? Injury 1991;22:29-31.

5. Cosan TE, Arslantas A, Guner AI, et al. Injury caused by deeply penetrating knife blade lodged in infratemporal fossa. Eur J Emerg Med 2001;8:51-4.

\section{Giant Eccrine Hidradenoma Mimicking a Malignant Tumor of the Salivary Gland}

Seung Hyun Oh, Chung Hun Kim, Euna Hwang

Department of Plastic and Reconstructive Surgery, CHA Bundang Medical Center, CHA University, Seongnam, Korea

\author{
Correspondence: Euna Hwang \\ Department of Plastic and Reconstructive Surgery, CHA Bundang Medical \\ Center, CHA University, 59 Yatap-ro, Bundang-gu, Seongnam 463-712, Korea \\ Tel: +82-31-780-5280, Fax: +82-31-780-5285 \\ E-mail: pshwang@cha.ac.kr \\ This article was presented at the 3rd Research and Reconstructive Forum on May 9-10, \\ 2013, in Daegu, Korea. \\ No potential conflict of interest relevant to this article was reported. \\ Received: 5 Aug 2013 • Revised: 25 Sep $2013 \bullet$ Accepted: 4 Oct 2013 \\ pISSN: 2234-6163• elSSN: 2234-6171 \\ http://dx.doi.org/10.5999/aps.2014.41.4.437 • Arch Plast Surg 2014;41:437-439 \\ Copyright (C) 2014 The Korean Society of Plastic and Reconstructive Surgeon \\ This is an Open Access article distributed under the terms of the Creative Commons \\ Attribution Non-Commercial License (http://creativecommons.org/licenses/by-nc/3.0/) \\ which permits unrestricted non-commercial use, distribution, and reproduction in any \\ medium, provided the original work is properly cited.
}

Eccrine hidradenoma is a rare benign cutaneous neoplasm, believed to arise from the distal excretory duct of the eccrine sweat glands [1]. Because a consensus has not been reached regarding its histogenesis, eccrine hidradenoma is also known as nodular hidradenoma, clear cell hidradenoma, eccrine acrospiroma, and eccrine sweat gland adenoma. It usually manifests as an asymptomatic, solitary, firm, cutaneous nodule, measuring between $5 \mathrm{~mm}$ and 20 $\mathrm{mm}$ in diameter. The tumor is usually confined to the dermis or subcutaneous layer without involving the deep structures. It occurs commonly in middle-aged and older adults, and can be found anywhere on the body without predilection for a particular site [2]. Its growth is slow, and a malignant change is rare. Several reports of giant eccrine hidradenoma can be found in the literature $[3,4]$. However, a case of a benign giant eccrine hidradenoma infiltrating the deeper structures beyond the muscle layer to the cranial base has not been reported thus far. Herein, we report a case of a benign giant nodular eccrine hidradenoma in the preauricular area that had infiltrated deep structures and extended into the cranial base.

An 81-year-old man presented with a firm fleshcolored, non-tender, lobulated mass measuring 8 $\mathrm{cm} \times 5 \mathrm{~cm}$ in the right preauricular area. He had difficulty opening his mouth and closing his right eyelid. A physical examination revealed a loss of forehead wrinkles, eyebrow ptosis, mild ectropion of the lower eyelid, and mild depression of the mouth corner on the right side of his face, which were consistent with facial nerve paralysis (HouseBrackman grade IV) (Fig. 1). The mass first appeared approximately 30 years ago, and the patient previously had the mass surgically excised at another clinic approximately 5 years previously. The tumor had slowly grown again over the last couple of years. Laboratory findings including all hematologic and biochemical examinations showed no abnormalities. A computed tomography (CT) scan revealed a large mass occupying the parotid space, masticatory space, and temporal region with infiltration into the temporalis muscle. The tumor mass compressed the internal jugular vein, displaced the external carotid artery inferiorly, and extended to the cranial base around the right foramen ovale without intracranial extension (Fig. 2). In the magnetic resonance imaging (MRI) scan, this heterogeneous tumor showed

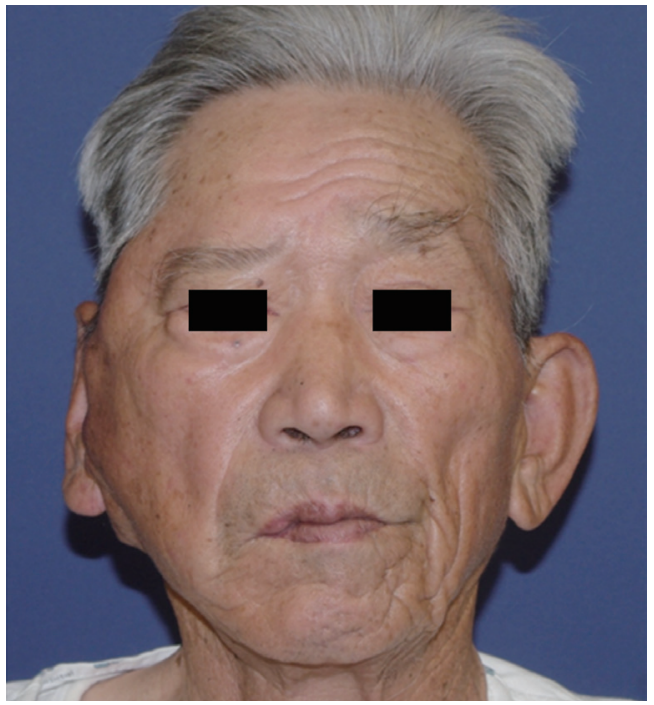

Fig. 1

A nodulated firm mass in the right preauricular area measuring $8 \mathrm{~cm} \times 5 \mathrm{~cm}$. The patient showed symptoms and signs of facial nerve paralysis on the right hemiface. 


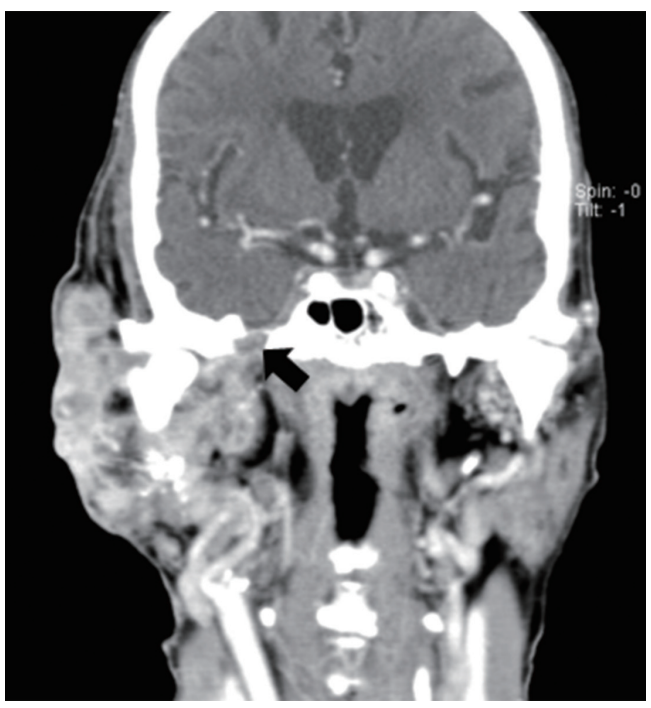

Fig. 2.

Computed tomography showed a large mass occupying the parotid space, the masticatory space, and the temporal region, with infiltrations into the temporalis muscle and pterygoid muscles. The tumor mass extended to the cranial base around the right foramen ovale without any intracranial extension (black arrow).

homogeneous enhancement and focal internal calcifications. Although CT and MRI findings indicated a malignancy of the salivary gland invading deep structures with regional metastasis, the result of a preliminary excisional biopsy of this tumor was consistent with a benign eccrine hidradenoma.

Although it was difficult to secure clear resection around the cranial base because of the poor accessibility and technical difficulty, surgery was planned to preserve the function of major neurovascular structures involved with the tumor. Because the mass was enveloped by a thick fibrotic capsule and severely adhered to adjacent tissues (sternocleidomastoid muscle, masseter muscle, and parotid gland), we could not preserve the facial nerve. The tumor was resected en bloc, including the overlying skin, facial nerve trunk, parotid gland with the invaded parts of the masseter muscle, and medial and lateral pterygoid muscles through the window created by temporary segmental mandibulectomy. Three separate daughter masses in the temporal region, which was impressed as lymph node metastasis, were removed. Nonetheless, a small amount of tumor near the foramen ovale remained. A frozen histologic examination showed no evidence of malignancy. The dead space that developed due to tumor removal was filled using a temporalis muscle flap.

A permanent histopathological examination was consistent with a nodular eccrine hidradenoma. The examination of a cut section showed that the mass had a grayish white gritty surface with a central hemorrhagic area (Fig. 3). The histopathological examination of the specimen revealed a multi-lobular tumor, which was composed of cellular masses separated by thin vacuolar connective tissue stroma. This cell mass showed two distinct cell populations. Most of them were large and round cells with a clear cytoplasm, owing to glycogen content. We also observed cells with a granular, faintly eosinophilic cytoplasm with round or oval nuclei. Cystic structures in the tumor were lined by cuboidal cells, and the lumens were filled with a faintly eosinophilic granular material (Fig. 4). No atypical mitotic figure was found, and the proliferation index was low (2\%), as assessed by Ki-67 immunohistochemistry. On the basis of these findings, the diagnosis of the nodular eccrine hidradenoma was confirmed. The patient continues to be closely observed for any evidence of regrowth from the remaining tumor mass. We chose a simple method for the reconstructive method of the facial paralysis, considering the patient's old age. At postoperative 3 weeks, we performed a static sling procedure with a prolene suture (\#2) and Endotine Ribbon (Coapt Systems Inc., Palo Alto, CA, USA) for the correction of the drooping soft tissue and the mouth corner of the ipsilateral hemiface. The sixmonth follow-up visit revealed no evidence of tumor regrowth (Fig. 5).

A review of previous reports indicated that giant eccrine hidradenoma extends from the main lesion and commonly reaches the subcutaneous tissue; however, the lesion seldom invades deep structures beyond the deep fascia. 


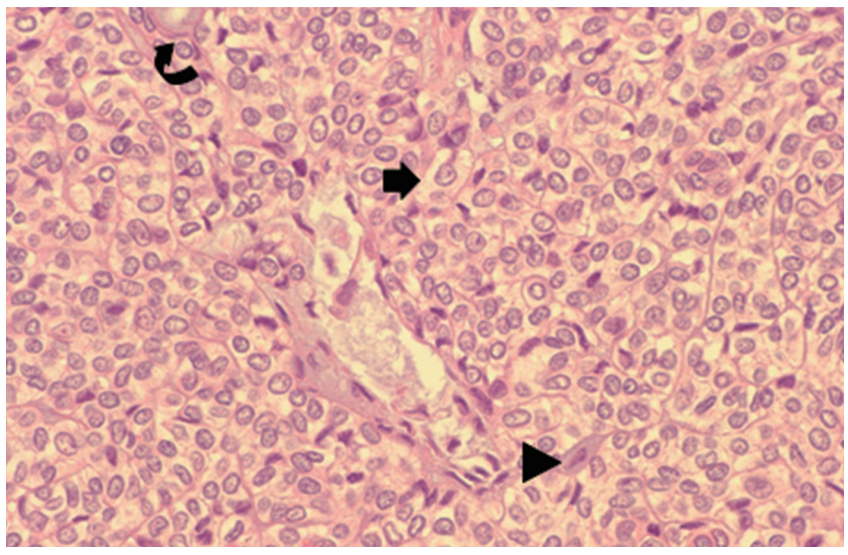

Fig. 4 .

Cell mass showing two distinct cell populations. Most cells were large and round with a clear cytoplasm (black arrow), owing to the glycogen content. Other cells had a granular, faintly eosinophilic cytoplasm with round or oval nuclei (black arrowhead). Ductal differentiation was present (black curved arrow) $\left(H \& E_{1} \times 200\right)$.

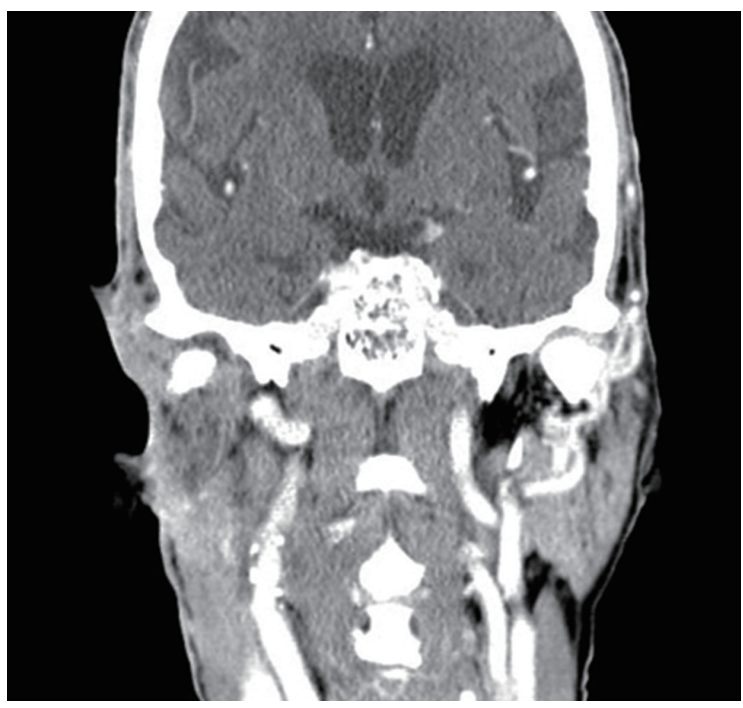

Fig. 5.

Computed tomography scan at 6 months after surgery.
In this case, the patient presented with a unilateral paralysis of the facial nerve. The tumor extended into the temporalis muscle under the zygomatic arch and the masticatory space, with infiltration into the medial and lateral pterygoid muscles. Further, there was a lack of circumscription, deep extension, infiltrating growth pattern, perineural invasion, and regional metastasis of the tumor mass. These findings were consistent with a malignant tumor of the salivary gland.

However, a histopathological examination revealed that the mass was a benign eccrine hidradenoma with a low proliferation index (2\%), as assayed by Ki-67 immunohistochemistry, and no cellular atypia.

Differentiation between pleomorphic adenoma and eccrine hidradenoma is not easy. Eccrine hidradenoma is typically characterized by two types of cells: eosinophilic cells and clear cells, which line duct-like structures. In contrast, pleomorphic adenoma is highly variable in appearance, even within individual tumors. In general, it is biphasic and is characterized by an admixture of polygonal epithelial and spindle-shaped myoepithelial elements in a background stroma.

Malignant transformation is extremely rare, and eccrine hidradenoma does not have a characteristic clinical appearance; therefore, differential diagnosis from other neoplasms, either benign or malignant, is only possible after a histopathological evaluation.

Although the exact mechanism of invasion by benign tumors has not yet been elucidated, benign tumor cells might invade into the deep structures along nerves such as the facial nerve and trigeminal nerve, as observed in this case. One report explained the mechanism of metastasis of benign tumors: the classical metastatic properties of malignant cells are the consequence of genetic abnormalities; conversely, benign metastasis can be explained by a mechanical "squeezing" of neoplastic cells into the lymph vessels after tumor overgrowth and/or trauma [5]. We speculate that the tumor cells might have migrated along the lymphatics and/or by neurovascular permeation and formed a remote mass separated from the main tumor. Therefore, benign eccrine hidradenoma has a tendency to show features of a malignant tumor, such as regional metastasis and infiltration of the deep structures.

\section{References}

1. Winkelmann RK, Wolff K. Solid-cystic hidradenoma of the skin: clinical and histopathologic study. Arch Dermatol 1968;97:651-61.

2. Laws RA, English JC 3rd, Elston DM. Acrospiroma: a case report and review. Cutis 1996;58:349-51.

3. Hunt SJ, Santa Cruz DJ, Kerl H. Giant eccrine acrospiroma. J Am Acad Dermatol 1990;23:663-8.

4. Demirci GT, Atis G, Altunay IK, et al. A giant benign clear cell hidradenoma on the anterior trunk. Dermatol Rep 2011;3:e45.

5. Stefanato CM, Ferrara G, Chaudhry IH, et al. Clear cell nodular hidradenoma involving the lymphatic system: a tumor of uncertain malignant potential or a novel example of "metastasizing" benign tumor? Am J Surg Pathol 2012;36:1835-40. 